\title{
Engineering Non-cellulosic Polysaccharides of Wood for the Biorefinery
}

\author{
Evgeniy Donev ${ }^{1}$, Madhavi Latha Gandla², Leif J. Jönsson ${ }^{2}$ and Ewa J. Mellerowicz ${ }^{1 *}$ \\ ' Department of Forest Genetics and Plant Physiology, Swedish University of Agricultural Sciences, Umeå, Sweden, \\ ${ }^{2}$ Department of Chemistry, Umeå University, Umeå, Sweden
}

\section{OPEN ACCESS}

Edited by:

Ronald Ross Sederoff,

North Carolina State University,

United States

Reviewed by:

Jozef Mravec,

University of Copenhagen, Denmark Rosemary White,

Commonwealth Scientific and Industrial Research Organisation (CSIRO), Australia

${ }^{*}$ Correspondence:

Ewa J. Mellerowicz

ewa.mellerowicz@s/u.se

Specialty section:

This article was submitted to

Plant Biotechnology,

a section of the journal

Frontiers in Plant Science

Received: 13 July 2018

Accepted: 28 September 2018

Published: 23 October 2018

Citation:

Donev E, Gandla ML, Jönsson LJ and Mellerowicz EJ (2018) Engineering Non-cellulosic Polysaccharides of Wood for the Biorefinery.

Front. Plant Sci. 9:1537. doi: 10.3389/fp/s.2018.01537
Non-cellulosic polysaccharides constitute approximately one third of usable woody biomass for human exploitation. In contrast to cellulose, these substances are composed of several different types of unit monosaccharides and their backbones are substituted by various groups. Their structural diversity and recent examples of their modification in transgenic plants and mutants suggest they can be targeted for improving wood-processing properties, thereby facilitating conversion of wood in a biorefinery setting. Critical knowledge on their structure-function relationship is slowly emerging, although our understanding of molecular interactions responsible for observed phenomena is still incomplete. This review: (1) provides an overview of structural features of major non-cellulosic polysaccharides of wood, (2) describes the fate of non-cellulosic polysaccharides during biorefinery processing, (3) shows how the non-cellulosic polysaccharides impact lignocellulose processing focused on yields of either sugars or polymers, and (4) discusses outlooks for the improvement of tree species for biorefinery by modifying the structure of non-cellulosic polysaccharides.

Keywords: non-cellulosic polysaccharides, woody biomass, secondary cell wall, hemicellulose, pectin, galactan, tree genetic improvement, wood biorefining

\section{NON-CELLULOSIC POLYSACCHARIDES IN WOOD}

Plant cell walls constitute the most abundant renewable resource on Earth (Pauly and Keegstra, 2008). Wood, which essentially consists of cell walls, is naturally degradable and renewable, and technologies are currently being developed aiming at utilization of all organic wood components, i.e., cellulose, lignin, non-cellulosic polysaccharides, and extractives. Non-cellulosic polysaccharides, which include hemicelluloses, pectins, type II arabinogalactan (AG-II), and callose, account for roughly one third of wood dry weight. Among these four groups, hemicelluloses are most abundant contributing to $26-33 \%$ of the dry weight in softwoods (conifer species) and 19$34 \%$ in hardwoods (dicotyledonous species) depending on species, cell type, developmental stage, and environmental conditions (Sjöström, 1993).

Non-cellulosic polysaccharides exhibit remarkable variability in different layers of wood cell walls, and chiefly define these layers (Mellerowicz and Gorshkova, 2012). Thus, the middle lamella

Abbreviations: AG-II, type II arabinogalactan; CML, compound middle lamella; GGM, galactoglucomannan; G-layer, gelatinous layer; GM, glucomannan; GT, glycosyltransferase family; GX, glucuronoxylan; HG, homogalacturonan; IRX, irregular xylem; KD, knock-down; LCCs, lignin carbohydrate complexes; PCW, primary cell wall; RES, reducing end sequence in xylan; RG-I, rhamnogalacturonan I; RG-II, rhamnogalacturonan II; S/G, syringyl/guaiacyl; SCW, secondary cell wall; Xylp, xylopyranosyl residue. 
is dominated by pectins, the primary cell wall (PCW) layer by pectins and xyloglucan, the secondary cell wall (SCW) layers by xylans and mannans, and the gelatinous layer (G-layer) present as a tertiary layer in tension wood fibers by galactans and AG-II. These different polysaccharides blend with the lignin matrix and cellulose microfibrils, and are involved in covalent, ionic, and hydrophobic interactions with other cell wall components and with themselves (Cosgrove, 2005; Scheller and Ulvskov, 2010). They are the main source of lignin carbohydrate complexes (LCCs) linking to lignin by ether, glycoside, or ester bonds (Lawoko and Henriksson, 2006; Balakshin et al., 2007; Giummarella and Lawoko, 2016; Giummarella et al., 2016). Thereby, the non-cellulosic polysaccharides affect cell wall architecture, wood traits, and properties of lignocellulosic biomass being favorite targets for improving biomass properties (reviewed in Tavares et al., 2015; Damm et al., 2016; Marriott et al., 2016; Wang et al., 2016; Smith et al., 2017). They are also a precious source of large amounts of assimilated carbon for which clever applications are being sought (e.g., Zhao et al., 2015; Oinonen et al., 2016).

Among hemicelluloses, xylan, which includes the glucuronoxylan (GX) of hardwoods and the arabinoglucuronoxylan of softwoods (Figure 1), is a ubiquitous component of wood SCWs (Donaldson and Knox, 2012; Kim and Daniel, 2012). Approx. 60\% of the xylopyranosyl residue (Xylp) of hardwood xylan are mono- or di-acetylated (Teleman et al., 2000, 2002). The acetyl groups compete with glucuronic acid for Xylp position 2, and a decrease of one of these substituents usually leads to an increase of the other (Chong et al., 2014; Lee et al., 2014). Mannans, which include water-soluble galactoglucomannan (GGM) and water-insoluble glucomannan (GM) (Figure 1), are the most abundant hemicelluloses in softwood SCWs, whereas hardwood SCWs contain lower fractions of GM (Teleman, 2009). Xyloglucan (Figure 1) is localized in PCWs of hardwoods and softwoods (Bourquin et al., 2002; Donaldson and Knox, 2012; Kim and Daniel, 2013), where it may associate with hydrophobic cellulose surfaces or become entrapped inside cellulose fibrils (Park and Cosgrove, 2015).

Pectins, which include homogalacturonan (HG), rhamnogalacturonan I (RG-I), and rhamnogalacturonan II (RG-II) (Figure 1), are acidic polysaccharides. They constitute a large part of the middle lamella and PCW layers, jointly referred to as the compound middle lamella (CML) (Kim and Daniel, 2013). Reaction wood, such as tension wood of hardwood and compression wood of softwood, typically contains high mass fractions of $\beta$-1,4-galactans (Figure 1) presumably associated with RG-I.

Water-soluble softwood arabinogalactan, a variant of AG-II (Figure 1), is highly abundant in larch (25\%). Other softwoods and hardwoods contain small amounts of AG-II. AG-II may be covalently linked to xylan and pectin (Tan et al., 2013). Callose (Figure 1), or laricinan, accumulates in hardwoods and softwoods in response to damage and stress (Teleman, 2009). It is abundant in pits and between cavities of the inner S2 layer in compression wood (Hoffman and Timell, 1970; Chaffey and Barlow, 2002; Altaner et al., 2010; Zhang et al., 2016).

This review addresses the importance of the non-cellulosic polysaccharides in technological processes currently used in wood biorefining, and prospects of altering them in trees for obtaining either higher productivity or improved lignocellulose properties, like extractability or improved biochemical conversion to sugars.

\section{FATE OF NON-CELLULOSIC POLYSACCHARIDES DURING WOOD BIOREFINING}

Biorefining of wood includes the pulping (mechanical and chemical pulping, as well as combinations thereof), biochemical processes, and thermochemical processes. Mechanical pulping aims at high recovery of all major wood constituents, including non-cellulosic polysaccharides (Sjöström, 1993; Ek et al., 2009). In contrast, chemical pulping and subsequent bleaching steps are designed to target the lignin and preserve the cellulose, whereas the fate of the non-cellulosic polysaccharides is strongly dependent on the aim and the process technology, which can be Kraft (sulfate), sulfite, soda, or organosolv pulping (Sjöström, 1993; Ek et al., 2009). For example, dissolving pulp manufacture based on sulfite pulping, or, sometimes, Kraft pulping, aims at producing a cellulose of relatively high purity, which means that most of the non-cellulosic polysaccharides are degraded and removed together with the lignin. In many other processes, such as conventional Kraft pulping for manufacturing of paper products, preservation of hemicelluloses is beneficial, as the pulp yield will then be higher.

Biochemical conversion is typically based on saccharification of the polysaccharides using pretreatment and enzymatic hydrolysis. This creates monosaccharides, which are then refined further using microbial fermentation or chemical catalysis. The aim of the pretreatment is to make the cellulose susceptible to enzymatic hydrolysis, which otherwise would be too slow and provide too low sugar yields. Among many different pretreatment methods (Yang and Wyman, 2008; Sun et al., 2016; Jönsson and Martín, 2016), hydrothermal pretreatment under acidic conditions, with or without externally added acid and with or without the disruptive effect of steam explosion, is a common approach. Due to autohydrolysis and formation of carboxylic acids, which are derived mainly from the non-cellulosic polysaccharides, the process will be acidic even without externally added acid (Jönsson and Martín, 2016). The main target of hydrothermal pretreatment under acidic conditions is the hemicellulose. Cellulose and lignin are also affected, but typically to much lesser degree than hemicelluloses, which can be degraded almost quantitatively in well-optimized pretreatment (Wang et al., 2018). The severity of the hydrothermal pretreatment (time, temperature, and acidity) needs to be adapted to the feedstock. Softwoods require higher severity, whereas hardwoods can be processed using lower severity. Nevertheless, pressurized reactors and temperatures in the range $160-240^{\circ} \mathrm{C}$ (Sun et al., 2016) are typically used to create a pretreated material that is suitable for subsequent enzymatic saccharification.

Thermochemical conversion processes, such as combustion, gasification, and pyrolysis, will degrade all organic wood constituents and are less relevant within the context of the current review. 


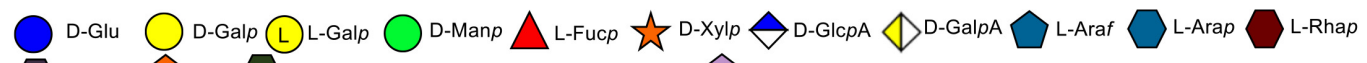
D-Dha $\square$ Apif $\bigcirc$ Kdo Ac O-acetyl MO-methyl BoBorate $\square$ Acef

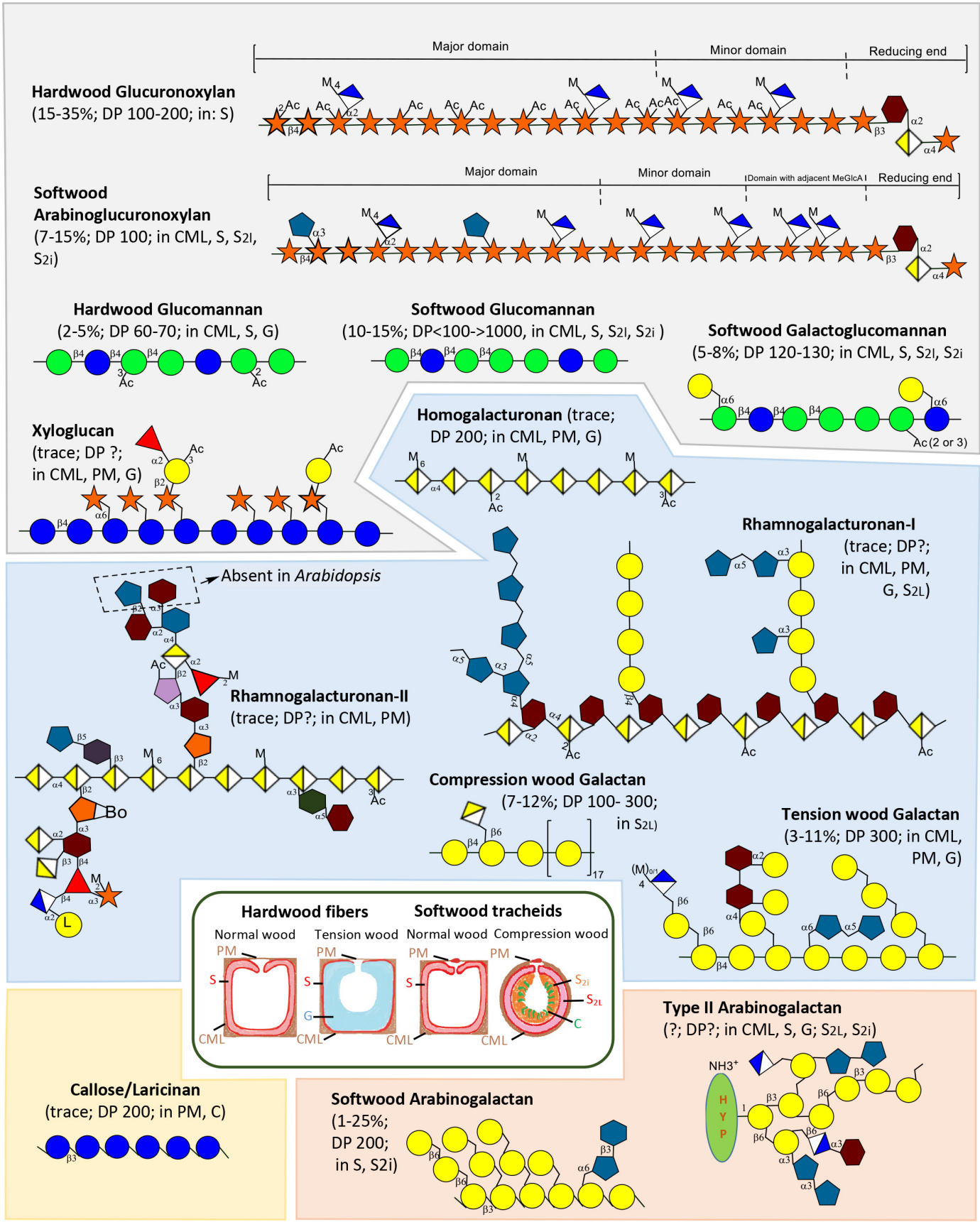

FIGURE 1 | Schematic illustration of types of non-cellulosic polysaccharides of wood, including hemicelluloses (gray background), pectins (blue background), callose (yellow background) and AGs-II (orange background), and hardwood fibers and softwood tracheids (inset). Polymer structures were based on different sources: hardwood GX (Teleman, 2009; Smith et al., 2017), softwood arabinoglucuronoxylan (Teleman, 2009; Martínez-Abad et al., 2017; Smith et al., 2017), hardwood and softwood glucomannan (GM), softwood GGM, tension and compression wood galactans, callose (Teleman, 2009), xyloglucan (Carpita and McCann, 2000; Teleman, 2009), HG (Atmodjo et al., 2013), RG-I and -II (Edashige and Ishii, 1996, 1997, 1998; Atmodjo et al., 2013), AG-II (Carpita and McCann, 2000; Hijazi et al., 2014), softwood arabinogalactan (Ponder and Richards, 1997; Teleman, 2009). Polymer localization is based on the following sources: hardwood GX and mannans (Kim and Daniel, 2012; Gorshkova et al., 2015; Guedes et al., 2017), softwood arabinoglucuronoxylan (Altaner et al., 2010; Donaldson and Knox, 2012), callose (Altaner et al., 2010; Zhang et al., 2016), xyloglucan (Bourquin et al., 2002; Sandquist et al., 2010; Nishikubo et al., 2011; Donaldson and Knox, 2012; Kim and Daniel, 2013; Guedes et al., 2017), HG (Kim and Daniel, 2013), RG-I /compression wood galactan/tension wood galactan (Gorshkova et al., 2015; Zhang et al., 2016; Guedes et al., 2017), AG-II/softwood arabinogalactan (Altaner et al., 2010; Guedes et al., 2017). PM, pit membrane; CML, compound middle lamella; S, secondary wall layer (S-layer), G, gelatinous layer (G-layer); C, cavities; $\mathrm{S}_{2 i}$, inner $\mathrm{S}_{2}$ layer; $\mathrm{S}_{2 \mathrm{~L}}$, outer lignified $\mathrm{S}_{2}$ layer. 


\section{ROLE OF NON-CELLULOSIC POLYSACCHARIDES IN RECALCITRANCE AND ATTEMPTS TO IMPROVE CONVERTIBILITY}

\section{Improvement of Xylan Structure Xylan Content and Length Affect Saccharification and Plant Growth}

Xylan is a key factor of recalcitrance, mainly by reducing cellulose accessibility (Bura et al., 2009; De Martini et al., 2013), prompting efforts to reduce its content in hardwoods. Attempts have been made using Arabidopsis as a model, either by targeting the xylan synthase complex or the enzymes synthesizing the reducing end sequence (RES) (Smith et al., 2017). However, strong reductions in xylan content led to mechanical failure of vessels [the socalled irregular xylem phenotype (IRX)] and stem weakening. Subsequent work with Populus indicated that xylan content can be reduced by $5-50 \%$ by knocking down (KD) different xylan biosynthetic genes including GT47C (Lee et al., 2009), two GAUT12/GT8D paralogs (Lee et al., 2011; Li et al., 2011; Biswal et al., 2015), GT43A and GT43B (Lee et al., 2011), and GT43B along with GT43C clade genes (Ratke et al., 2018) or by expressing fungal xylanase $H v X y l 1$ and targeting it to cell walls (Kaida et al., 2009; Table 1). Such reductions either did not affect (Lee et al., 2009) or stimulated growth (Biswal et al., 2015; Ratke et al., 2018), decreased xylem cell wall thickness (Li et al., 2011; Ratke et al., 2018), which sometimes (Lee et al., 2009, 2011) was coupled to a mild IRX phenotype. Beside xylan, the cellulose content decreased in case of GT47C KD, coupled with increased lignification (Lee et al., 2009). A similar increase in lignin coupled with brittleness of stems was observed in strong KD GAUT121 and -2 (Li et al., 2011), but not when only GAUT12-1 was reduced (Biswal et al., 2015). Lignin syringyl/guaiacyl (S/G) ratio was increased in GAUT12-1 KD poplar (Biswal et al., 2015), and in severe KD GT43B poplar (Lee et al., 2011). However, the S/G ratio was reduced without change in lignin content in mildly affected GT43B and GT43C KD (Ratke et al., 2018). Thus, it is difficult to predict how lignin might be affected in transgenic lines with reduced xylan content, and these changes should be carefully monitored, since they affect saccharification.

For non-pretreated wood, downregulation of GT43 genes resulted in a $30 \%$ increase in glucose yield in enzymatic saccharification (Lee et al., 2011; Ratke et al., 2018), but the benefits were less apparent after acid pretreatment (Ratke et al., 2018; Table 1). Reductions in xylan content by downregulation of GAUT12/GT8D did not improve saccharification without pretreatment (Lee et al., 2011) or did so only slightly (after steam pretreatment) (Biswal et al., 2015) whereas post-synthetic xylan reduction resulted in approx. 50\% increased glucose yield in saccharification after steam pretreatment (Kaida et al., 2009; Table 1). There might be several and possibly opposing factors at play affecting saccharification. For example, cell wall thickness, lignin content and composition, and content of tension wood can all affect glucose yields (Escamez et al., 2017). KD GAUT12/GT8D poplar had less LCCs, which contribute to recalcitrance (Min et al., 2014a). Clearly, manipulation of xylan induces indirect effects, some of which, such as increased growth (Biswal et al., 2015; Derba-Maceluch et al., 2015; Yang et al., 2017; Ratke et al., 2018), or increased drought tolerance (Keppler and Showalter, 2010) are interesting for biotechnological applications.

\section{Xylan Acetylation Affects Cell Wall Architecture}

Deacetylation of lignocellulosic biomass prior to enzymatic saccharification results in improved sugar yields (reviewed by Pawar et al., 2013). For lignocellulosic biomass with high acetyl content such as hardwoods, reduction of acetylation might have an added benefit for ethanolic fermentation processes, as high concentrations of acetic acid are inhibitory to fermenting microorganisms (reviewed by Jönsson and Martín, 2016).

Modest reductions in acetylation in KD RWA aspen (Pawar et al., 2017b) and in aspen expressing fungal xylan acetyl esterase AnAXE1 targeted to cell walls (Pawar et al., 2017a; Table 1) were well tolerated by plants. These plants yielded $20-25 \%$ more glucose in enzymatic saccharification without pretreatment. Results with Arabidopsis (Pawar et al., 2016) suggested that deacetylation in planta reduces recalcitrance by other mechanism than reducing the inhibition of xylan enzymatic hydrolysis by acetyl groups. Indeed, aspen expressing $A n A X E 1$ exhibited increased lignin solubility and reduced xylan content, xylan chain length, and lignin S/G ratio (Pawar et al., 2017a). Increased extractability of lignin and xylan agrees with the suggested xylan models (Ruel et al., 2006; Busse-Wicher et al., 2014), where the minor xylan domain (Figure 1) interacts with lignin. This domain, characterized by consecutive Xylp acetylation, would become (after deacetylation) more susceptible to hydrolysis by wall-residing enzymes, such as XYN10A (Derba-Maceluch et al., 2015), leading to loosening of xylan fraction interacting with lignin.

Overexpression of PdDUF231A, similar to AtPMR5, in $P$. deltoides resulted in increased xylan acetylation and surprisingly in improved saccharification without pretreatment (Yang et al., 2017; Table 1). Decreased lignin content and increased cellulose content in transgenic lines might have affected the sugar yield.

\section{Significance of Glucuronosylation}

Glucuronosylation of xylan makes its backbone less prone to hydrolysis by $\mathrm{GH} 10$ and GH11 xylanases, and requires $\alpha$-glucuronidases for saccharification (Mortimer et al., 2010). It is also associated with LCCs of hardwoods (Min et al., 2014a,b; Bååth et al., 2016). The majority of glucuronate in SCW xylan is methylated (Teleman, 2009) and KD of a GX methyl transferases DUF579-3/GXM3 in poplar reduced not only methylation but also resulted in reduced xylan glucuronosylation, and reduced growth (Song et al., 2016). Xylose yield of acid pretreatment increased as well as glucose yield of enzymatic saccharification. Thus, methylation of glucuronate is a promising target, but some means of avoiding growth penalty need to be designed.

To reduce ester links between GX and lignin in aspen, a fungal glucuronoyl esterase was expressed and targeted to cell walls (Gandla et al., 2015; Table 1). Increased cellulose-to-glucose conversion was observed, but plants exhibited premature leaf senescence and impaired growth (Gandla et al., 2015). There 


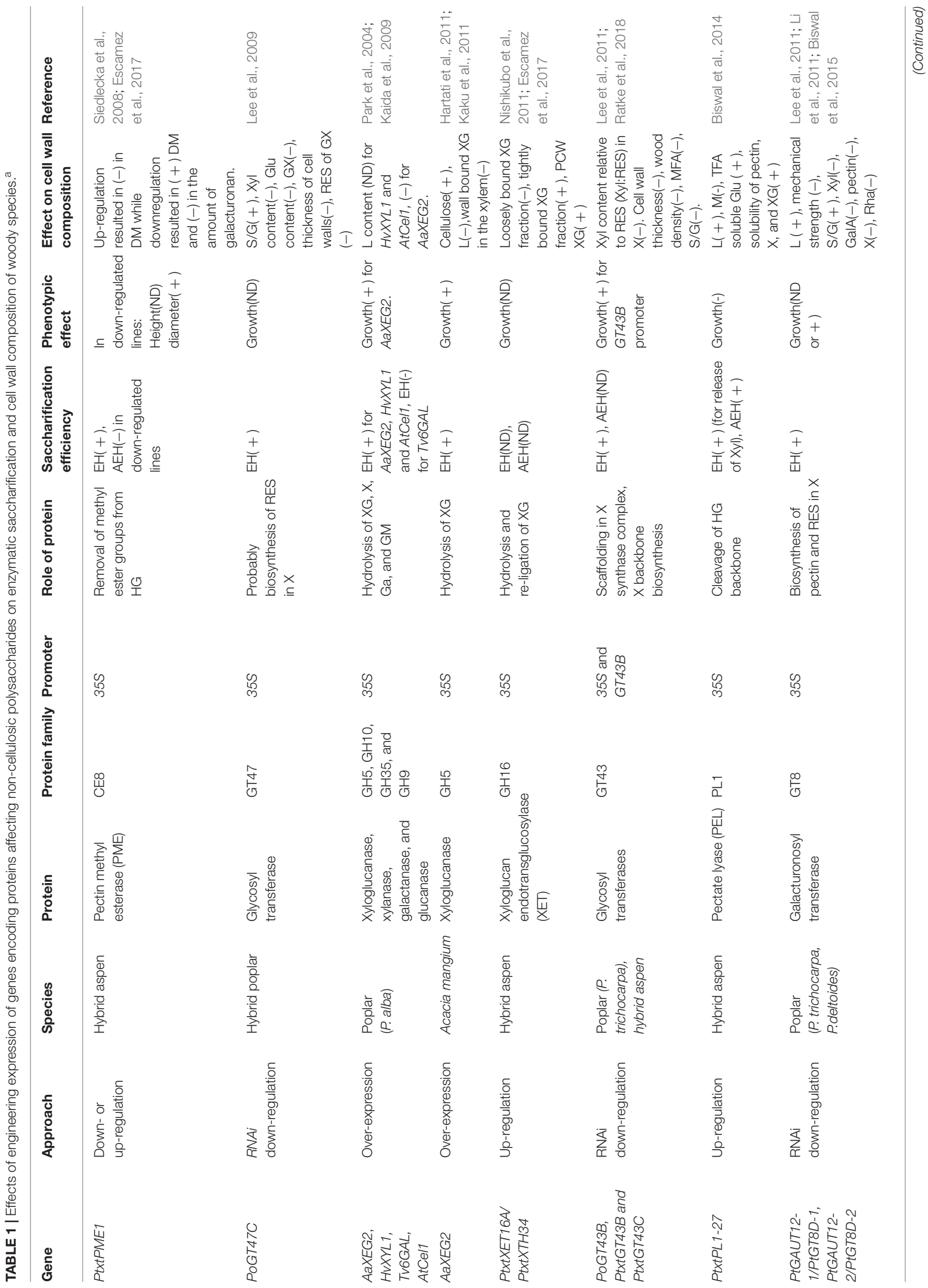







was a decrease in extractives and an increase in lignin (Gandla et al., 2015) and mechanisms underlying these responses are not understood.

\section{Prospects for Mannan Structure Improvement}

GGM is tightly associated with cellulose microfibrils (Schröder et al., 2009; Salmén, 2015) and increasing its extractability could be beneficial for saccharification. Mannan hydrolysis in planta by overexpression of plasma-membrane-bound mannanase MAN6 induced production of active galactoglucomannooligosaccharides that modified growth of poplar and inhibited SCW formation (Zhao et al., 2013), making this approach problematic. However, overexpression of extracellular GM-active endoglucanase AtCel1 in poplar lead to approx. 30\% increase in sugar yield and in cellulose conversion in saccharification after steam pretreatment (Kaida et al., 2009).

Mannan biosynthetic genes identified to date include a GM synthase (CSLA clade) (Suzuki et al., 2006), a mannan galactosyltransferase (GT34) (Edwards et al., 2004), and unknown function GT65 members AtMSR1 and 2 (Wang et al., 2013), and could be employed for biomass modification. For example, increasing the degree of galactosylation of mannan by increasing expression of mannan galactosyltransferase might lead to higher solubility of GGM (Edwards et al., 2004).

\section{Xyloglucan and Pectins - Minor Wood Components With High Impact on \\ Properties}

Several lines of evidence indicate that xyloglucan and pectins, despite being minor wood components (below 5\% dry weight), have significant effects on wood properties, including recalcitrance. Increased xyloglucan content in aspen obtained by overexpressing XTH34/XET16A did not affect growth (Nishikubo et al., 2011) nor saccharification (Escamez et al., 2017), but its reduction in poplar, achieved by expression of fungal xyloglucanase AaXEG2, highly stimulated growth, wood cellulose content, density, and mechanical strength in the greenhouse-grown poplar (Park et al., 2004; Table 1). Cellulose microfibrils were larger in transgenic plants (Yamamoto et al., 2011) and their lignocellulose yielded almost $50 \%$ more glucose per wood weight and per cellulose weight in saccharification after steam pretreatment (Kaida et al., 2009). Similar effects on growth and saccharification were observed in Acacia mangium (Kaku et al., 2011; Hartati et al., 2011). However, in a 4-year field trial transgenic poplars expressing AaXEG2 displayed substantially reduced biomass (Taniguchi et al., 2012). Furthermore, the plants were unable to bend upward when placed horizontally despite forming tension wood as in wild type, suggesting that xyloglucan is essential for generation of tensile stress (Baba et al., 2009).

Suppression of aspen pectin methyl esterase PME1 resulted in highly methylesterified HG in developing wood (Siedlecka et al., 2008; Table 1). The glucose yields in saccharification without pretreatment increased, but there was no improvement after acid pretreatment (Escamez et al., 2017).
Overexpression of aspen wood-expressed pectate lyase PL127 increased the solubility of xylan and xyloglucan suggesting that HG constrains the solubility of main non-cellulosic polysaccharides (Biswal et al., 2014; Table 1). There was a positive effect on the glucose yield for the transgenic lines, but only after acid pretreatment. Interestingly, decreased HG biosynthesis in poplar by $\mathrm{KD}$ of the GAUT genes involved in biosynthesis of pectin and xylan (Mohnen, 2008), led to substantial growth stimulation and a small increase in glucose yields in saccharification after acid or hot-water pretreatment (Biswal et al., 2015; Biswal et al., 2018; Table 1).

\section{FUTURE PROSPECTS FOR IMPROVING NON-CELLULOSIC POLYSACCHARIDES FOR BIOREFINING OF WOOD}

Two decades of research on modifying non-cellulosic polysaccharides have provided some insight on the role of these polysaccharides in cell wall architecture, on their importance for the efficiency of pretreatment and enzymatic saccharification, and have identified some off-target effects. They have also identified the most promising targets for achieving better growth and saccharification. Most research has been focused on xylan modification identifying xylan chain length, degree of acetylation, glucuronosylation, and glucuronosyl methylation as possible targets. The discovery of microbial enzymes hydrolyzing ester links between glucuronosyl units and lignin opened new prospects for directly reducing LCCs in cell walls, and should be further explored. The modification of minor wood components, HG and xyloglucan, had some of the highest impacts on saccharification, pointing to the crucial role of these polymers in wood integrity, but their modification sometimes led to growth inhibition. Using the wood-specific promoters, such as the GT43B promoter (Ratke et al., 2015), for expressing transgenes, can prevent off-target modifications in meristems, root hairs and other primary walled tissues, and possibly avoid growth penalty. Overall, there were few attempts in trees to employ different promoters for expressing the transgenes. Utilization of heat inducible promoters and heat-active enzymes in trees for modifying properties during post-harvest heat treatment has not yet been explored. Modification of other non-cellulosic components, for example mannans, RG-I and RG-II has not been so far investigated in trees and will be interesting to reveal the role of these polymers in woody biomass organization.

Currently, there is little understanding of the molecular mechanisms responsible for the observed phenotypes. In most cases, the distinction between primary and secondary effects of transgene expression is not possible. Interestingly, some types of xylan modification lead to increased growth (Biswal et al., 2015; Derba-Maceluch et al., 2015; Ratke et al., 2018), which might be mediated by the SCW integrity sensing mechanism (Ratke et al., 2018). On the other hand, the transcriptome analyses in Arabidopsis mutants impaired in xylan biosynthesis did not reveal any changes indicative of SCW integrity sensing (Faria-Blanc et al., 2018). It would be important to elucidate if such signaling 
exists and if so, what triggers it, for successful modification of SCW.

Almost all results reviewed here are based on greenhouse experiments. Experience with xyloglucanase-modified poplar (Taniguchi et al., 2012) points to a need for early field experiments to pinpoint possible problems of transgenic lines. Field-grown trees will also provide sufficient biomass for testing pulping properties.

Finally, the tension wood of hardwoods appears to be particularly suitable for saccharification (Brereton et al., 2012; Sawada et al., 2018). Progress in identification of pathways involved in the induction of tension wood (Felten et al., 2018) will make it possible to design strategies to stimulate tension wood formation

\section{REFERENCES}

Altaner, C. M., Tokareva, E. N., Jarvis, M. C., and Harris, P. J. (2010). Distribution of $(1 \rightarrow 4)-\beta$-galactans, arabinogalactan proteins, xylans and $(1 \rightarrow 3)-\beta$-glucans in tracheid cell walls of softwoods. Tree Physiol. 30, 782-793. doi: 10.1093/ treephys/tpq021

Atmodjo, M. A., Hao, Z., and Mohnen, D. (2013). Evolving views of pectin biosynthesis. Annu. Rev. Plant Biol. 64, 747-779. doi: 10.1146/annurev-arplant042811-105534

Bååth, J., Giummarella, N., Klaubauf, S., Lawoko, M., and Olsson, L. (2016). A glucuronoyl esterase from Acremonium alcalophilum cleaves native lignincarbohydrate ester bonds. FEBS Lett. 590, 2611-2618. doi: 10.1002/1873-3468

Baba, K., Park, Y. W., Kaku, T., Kaida, R., Takeuchi, M., Yoshida, M., et al. (2009). Xyloglucan for generating tensile stress to bend tree stem. Mol. Plant 2, 893-903. doi: $10.1093 / \mathrm{mp} / \mathrm{ssp} 054$

Balakshin, M., Capanema, E. A., and Chang, H. (2007). MWL fraction with a high concentration of lignin-carbohydrate linkages: isolation and 2D NMR spectroscopic analysis. Holzforschung 61, 1-7. doi: 10.1515/HF.2007.001

Biswal, A. K., Atmodjo, M. A., Li, M., Baxter, H. L., Yoo, C. G., Pu, Y., et al. (2018). Sugar release and growth of biofuel crops are improved by downregulation of pectin biosynthesis. Nat. Biotechnol. 36, 249-257. doi: 10.1038/nbt.4067

Biswal, A. K., Hao, Z., Pattathil, S., Yang, X., Winkeler, K., Collins, C., et al. (2015). Downregulation of GAUT12 in Populus deltoides by RNA silencing results in reduced recalcitrance, increased growth and reduced xylan and pectin in a woody biofuel feedstock. Biotechnol. Biofuels 18:41. doi: 10.1186/s13068-0 15-0218-y

Biswal, A. K., Soeno, K., Gandla, M. L., Immerzeel, P., Pattathil, S., Lucenius, J., et al. (2014). Aspen pectate lyase PtxtPL1-27 mobilizes matrix polysaccharides from woody tissues and improves saccharification yield. Biotechnol. Biofuels 7:11. doi: 10.1186/1754-6834-7-11

Bourquin, V., Nishikubo, N., Abe, H., Brumer, H., Denman, S., Eklund, M., et al. (2002). Xyloglucan endotransglycosylases have a function during the formation of secondary cell walls of vascular tissues. Plant Cell 14, 3073-3088. doi: 10. $1105 /$ tpc.007773

Brereton, N. J., Ray, M. J., Shield, I., Martin, P., Karp, A., and Murphy, R. J. (2012). Reaction wood - A key cause of variation in cell wall recalcitrance in willow. Biotechnol. Biofuels 5:83. doi: 10.1186/1754-6834-5-83

Bura, R., Chandra, R., and Saddler, J. (2009). Influence of xylan on the enzymatic hydrolysis of steam-pretreated corn stover and hybrid poplar. Biotechnol. Prog. 25, 315-322. doi: 10.1002/btpr.98

Busse-Wicher, M., Gomes, T. C. F., Tryfona, T., Nikolovski, N., Stott, K., Grantham, N. J., et al. (2014). The pattern of xylan acetylation suggests xylan may interact with cellulose microfibrils as a twofold helical screw in the secondary plant cell wall of Arabidopsis thaliana. Plant J. 79, 492-506. doi: $10.1111 /$ tpj. 12575

Carpita, N., and McCann, M. (2000). "The Cell Wall," in Biochemistry and Molecular Biology of Plants, eds B. B. Buchanan, W. Gruissem, and R. L. Jones (Rockville, MD: American Society of Plant Biologists), 52-108. in normal growth conditions for dedicated biorefinery feedstocks.

\section{AUTHOR CONTRIBUTIONS}

ED, LJJ, and EJM wrote the paper. MLG prepared the Figure and the Table. All authors read and approved the final submission.

\section{FUNDING}

SSF project ValueTree, the Swedish Energy Agency, and the Bio4Energy program (www.bio4energy.se).

Chaffey, N., and Barlow, P. (2002). Myosin, microtubules, and microfilaments: cooperation between cytoskeletal components during cambial cell division and secondary vascular differentiation in trees. Planta 214, 526-536. doi: 10.1007/ s004250100652

Chong, S. L., Virkki, L., Maaheimo, H., Juvonen, M., Derba-Maceluch, M., Koutaniemi, S., et al. (2014). O-Acetylation of glucuronoxylan in Arabidopsis thaliana wild type and its change in xylan biosynthesis mutants. Glycobiology 24, 494-506. doi: 10.1093/glycob/cwu017

Cosgrove, D. J. (2005). Growth of the plant cell wall. Nat. Rev. Mol. Cell Biol. 6, 850-861. doi: 10.1038/nrm1746

Damm, T., Commandeur, U., Fischer, R., and Usadel, B. (2016). Improving the utilization of lignocellulosic biomass by polysaccharide modification. Process Biochem. 51, 288-296. doi: 10.1016/j.procbio.2015.12.003

De Martini, J. D., Pattathil, S., Miller, J. S., Li, H., Hahn, M. G., and Wyman, C. E. (2013). Investigating plant cell wall components that affect biomass recalcitrance in poplar and switchgrass. Energy Environ. Sci. 6, 898-909. doi: 10.1039/c3ee23801f

Derba-Maceluch, M., Awano, T., Takahashi, J., Lucenius, J., Ratke, C., Kontro, I., et al. (2015). Suppression of xylan endotransglycosylase PtxtXyn10A affects cellulose microfibril angle in secondary wall in aspen wood. New Phytol. 205, 666-681. doi: 10.1111/nph.13099

Donaldson, L. A., and Knox, J. P. (2012). Localization of cell wall polysaccharides in normal and compression wood of radiata pine: relationships with lignification and microfibril orientation. Plant Physiol. 158, 642-653. doi: 10.1104/pp.111. 184036

Edashige, Y., and Ishii, T. (1996). Pectic polysaccharides from xylem-differentiating zone of Cryptomeria japonica. Phytochemistry 42, 611-616. doi: 10.1016/00319422(95)00958-2

Edashige, Y., and Ishii, T. (1997). Rhamnogalacturonan I from xylem differentiating zones of Cryptomeria japonica. Carbohydr. Res. 304, 357-365. doi: 10.1016/S0008-6215(97)10042-8

Edashige, Y., and Ishii, T. (1998). Rhamnogalacturonan II from cell walls of Cryptomeria japonica. Phytochemistry 49, 681-690. doi: 10.1016/S0 031-9422(98)00238-6

Edwards, M. E., Choo, T., Dickson, C., Scott, C., Gidley, M. J., and Reid, J. S. G. (2004). The seeds of Lotus japonicus lines transformed with sense, antisense, and sense/antisense galactomannan galactosyltransferase constructs have structurally altered galactomannans in their endosperm cell walls. Plant Physiol. 134, 1153-1162. doi: 10.1104/pp.103.029967

Ek, M., Gellerstedt, G., and Henriksson, G. (2009). Pulping Chemistry and Technology. Berlin: De Gruyter. doi: 10.1515/9783110213423

Escamez, S., Gandla, L. M., Derba-Maceluch, M., Lundqvist, S. O., Mellerowicz, E. J., Jönsson, L. J., et al. (2017). A collection of genetically engineered Populus trees reveals wood biomass traits that predict glucose yield from enzymatic hydrolysis. Sci. Rep. 7:15798. doi: 10.1038/s41598-017-16013-0

Faria-Blanc, N., Mortimer, J. C., and Dupree, P. (2018). A transcriptomic analysis of xylan mutants does not support the existence of a secondary cell wall integrity system in Arabidopsis. Front. Plant Sci. 9:384. doi: 10.3389/fpls.2018.00384 
Felten, J., Vahala, J., Love, J., Gorzsás, A., Rüggeberg, M., Delhomme, N., et al. (2018). Ethylene signaling induces gelatinous layers with typical features of tension wood in hybrid aspen. New Phytol. 218, 999-1014. doi: 10.1111/nph. 15078

Gandla, L. M., Derba-Maceluch, M., Liu, X., Gerber, L., Master, E. R., Mellerowicz, E. J., et al. (2015). Expression of a fungal glucuronoyl esterase in Populus: effects on wood properties and saccharification efficiency. Phytochemistry 112, 210-220. doi: 10.1016/j.phytochem.2014.06.002

Giummarella, N., and Lawoko, M. (2016). Structural basis for the formation and regulation of lignin-xylan bonds in birch. ACS Sustain. Chem. Eng. 4, 5319-5326. doi: 10.1021/acssuschemeng.6b00911

Giummarella, N., Zhang, L., Henriksson, G., and Lawoko, M. (2016). Structural features of mildly fractionated lignin carbohydrate complexes (LCC) from spruce. RSC Adv. 6, 42120-42131. doi: 10.1039/c6ra02399a

Gorshkova, T., Mokshina, N., Chernova, T., Ibragimova, N., Salnikov, V., Mikshina, P., et al. (2015). Aspen tension wood fibers contain $\beta$-(1 $\rightarrow 4)$ galactans and acidic arabinogalactans retained by cellulose microfibrils in gelatinous walls. Plant Physiol. 169, 2048-2063. doi: 10.1104/pp.15.00690

Guedes, F. T. P., Laurans, F., Quemener, B., Assor, C., Lainé-Prade, V., Boizot, N., et al. (2017). Non-cellulosic polysaccharide distribution during G-layer formation in poplar tension wood fibers: abundance of rhamnogalacturonan I and arabinogalactan proteins but no evidence of xyloglucan. Planta 246, 857-878. doi: 10.1007/s00425-017-2737-1

Hartati, S., Sudarmonowati, E., Kaku, T., Ikegaya, H., Kaida, R., Baba, K., et al. (2011). Overexpression of xyloglucanase (AaXEG2) accelerates heteroblastic development in mangium leaves. J. Wood Sci. 57, 463-469. doi: 10.1007/s10 086-011-1211-0

Hijazi, M., Velasquez, S. M., Jamet, E., Estevez, J. M., and Albenne, C. (2014). An update on post-translational modifications of hydroxyproline-rich glycoproteins: toward a model highlighting their contribution to plant cell wall architecture. Front. Plant Sci. 5:395. doi: 10.3389/fpls.2014.00395

Hoffman, G. C., and Timell, T. E. (1970). Isolation of a $\beta$-1,3-glucan (laricinan) from compression wood of Larix laricina. Wood Sci. Technol. 4, 159-162. doi: 10.1007/BF00365301

Jönsson, L. J., and Martín, C. (2016). Pretreatment of lignocellulose: formation of inhibitory by-products and strategies for minimizing their effects. Bioresour. Technol. 199, 103-112. doi: 10.1016/j.biortech.2015.10.009

Kaida, R., Kaku, T., Baba, K., Oyadomari, M., Watanabe, T., Nishida, K., et al. (2009). Loosening xyloglucan accelerates the enzymatic degradation of cellulose in wood. Mol. Plant 2, 904-909. doi: 10.1093/mp/ssp060

Kaku, T., Kaida, R., Baba, K., Hartati, S., Sudarmonowati, E., and Hayashi, T. (2011). Improvement of fermentable sugar yields of mangium through transgenic overexpression of xyloglucanase. J. Wood Sci. 57, 545-548. doi: 10. 1007/s10086-011-1180-3

Keppler, B. D., and Showalter, A. M. (2010). IRX14 and IRX14-LIKE, two glycosyl transferases involved in glucuronoxylan biosynthesis and drought tolerance in Arabidopsis. Mol. Plant 3, 834-841. doi: $10.1093 / \mathrm{mp} / \mathrm{ssq} 028$

Kim, J. S., and Daniel, G. (2012). Distribution of glucomannans and xylans in poplar xylem and their changes under tension stress. Planta 236, 35-50. doi: $10.1007 / \mathrm{s} 00425-012-1588-\mathrm{Z}$

Kim, J. S., and Daniel, G. (2013). Developmental localization of homogalacturonan and xyloglucan epitopes in pit membranes varies between pit types in two poplar species. IAWA J. 34, 245-262. doi: 10.1163/22941932-00000021

Lawoko, M., and Henriksson, G. (2006). Characterisation of lignin-carbohydrate complexes ( LCCs ) of spruce wood (Picea abies L.) isolated with two methods. Holzforschung 60, 156-161. doi: 10.1515/HF.2006.025

Lee, C., Teng, Q., Huang, W., Zhong, R., and Ye, Z.-H. (2009). Downregulation of PoGT47C expression in poplar results in a reduced glucuronoxylan content and an increased wood digestibility by cellulase. Plant Cell Physiol. 50, 1075-1089. doi: $10.1093 / \mathrm{pcp} / \mathrm{pcp} 060$

Lee, C., Teng, Q., Huang, W., Zhong, R., and Ye, Z. H. (2011). Molecular dissection of xylan biosynthesis during wood formation in poplar. Mol. Plant 4, 730-747. doi: $10.1093 / \mathrm{mp} / \mathrm{ssr} 035$

Lee, C., Teng, Q., Zhong, R., and Ye, Z. H. (2014). Alterations of the degree of xylan acetylation in Arabidopsis xylan mutants. Plant Signal. Behav. 9:e27797. doi: $10.4161 /$ psb. 27797

Li, Q., Min, D., Wang, J. P., Peszlen, I., Horvath, L., Horvath, B., et al. (2011). Down-regulation of glycosyltransferase 8D genes in Populus trichocarpa caused reduced mechanical strength and xylan content in wood. Tree Physiol. 31, 226-236. doi: 10.1093/treephys/tpr008

Marriott, P. E., Gómez, L. D., and McQueen-Mason, S. J. (2016). Unlocking the potential of lignocellulosic biomass through plant science. New Phytol. 209, 1366-1381. doi: 10.1111/nph.13684

Martínez-Abad, A., Berglund, J., Toriz, G., Gatenholm, P., Henriksson, G., Lindström, M., et al. (2017). Regular motifs in xylan modulate molecular flexibility and interactions with cellulose surfaces. Plant Physiol. 175, 1579-1592. doi: 10.1104/pp.17.01184

Mellerowicz, E. J., and Gorshkova, T. A. (2012). Tensional stress generation in gelatinous fibres: a review and possible mechanism based on cell-wall structure and composition. J. Exp. Bot. 63, 551-565. doi: 10.1093/jxb/err339

Min, D. Y., Li, Q., Chiang, V., Jameel, H., Chang, H. M., and Lucia, L. (2014a). The influence of lignin-carbohydrate complexes on the cellulasemediated saccharification I: Transgenic black cottonwood (western balsam poplar, California poplar) $P$. trichocarpa including the xylan down-regulated and the lignin down-regulated lines. Fuel 119, 207-213. doi: 10.1016/j.fuel.2013. 11.047

Min, D. Y., Yang, C., Chiang, V., Jameel, H., and Chang, H. M. (2014b). The influence of lignin-carbohydrate complexes on the cellulase-mediated saccharification II: Transgenic hybrid poplars (Populus nigra L. and Populus maximowiczii A.). Fuel 116, 56-62. doi: 10.1016/j.fuel.2013.07.046

Mohnen, D. (2008). Pectin structure and biosynthesis. Curr. Opin. Plant Biol. 11, 266-277. doi: 10.1016/j.pbi.2008.03.006

Mortimer, J. C., Miles, G. P., Brown, D. M., Zhang, Z., Segura, M. P., Weimar, T., et al. (2010). Absence of branches from xylan in Arabidopsis gux mutants reveals potential for simplification of lignocellulosic biomass. Proc. Natl. Acad. Sci. U.S.A. 107, 17409-17414. doi: 10.1073/pnas.1005456107

Nishikubo, N., Takahashi, J., Roos, A. A., Derba-Maceluch, M., Piens, K., Brumer, H., et al. (2011). Xyloglucan endo-transglycosylase-mediated xyloglucan rearrangements in developing wood of hybrid aspen. Plant Physiol. 155, 399-413. doi: 10.1104/pp.110.166934

Oinonen, P., Krawczyk, H., Ek, M., Henriksson, G., and Moriana, R. (2016). Bioinspired composites from cross-linked galactoglucomannan and microfibrillated cellulose: thermal, mechanical and oxygen barrier properties. Carboh. Pol. 136, 146-153. doi: 10.1016/j.carbpol.2015.09.038

Park, Y. B., and Cosgrove, D. J. (2015). Xyloglucan and its interactions with other components of the growing cell wall. Plant Cell Physiol. 56, 180-194. doi: $10.1093 / \mathrm{pcp} / \mathrm{pcu} 204$

Park, Y. W., Baba, K., Furuta, Y., Iida, I., Sameshima, K., Arai, M., et al. (2004). Enhancement of growth and cellulose accumulation by overexpression of xyloglucanase in poplar. FEBS Lett. 564, 183-187. doi: 10.1016/S0014-5793(04) 00346- 1

Pauly, M., and Keegstra, K. (2008). Cell-wall carbohydrates and their modification as a resource for biofuels. Plant J. 54, 559-568. doi: 10.1111/j.1365-313X.2008. 03463.x

Pawar, P. M. A., Derba-Maceluch, M., Chong, S. L., Gandla, M. L., Bashar, S. S., Sparrman, T., et al. (2017a). In muro deacetylation of xylan affects lignin properties and improves saccharification of aspen wood. Biotechnol. Biofuels 10:98. doi: 10.1186/s13068-017-0782-4

Pawar, P. M. A., Derba-Maceluch, M., Chong, S. L., Gómez, L. D., Miedes, E., Banasiak, A., et al. (2016). Expression of fungal acetyl xylan esterase in Arabidopsis thaliana improves saccharification of stem lignocellulose. Plant Biotechnol. J. 14, 387-397. doi: 10.1111/pbi.12393

Pawar, P. M. A., Koutaniemi, S., Tenkanen, M., and Mellerowicz, E. J. (2013). Acetylation of woody lignocellulose: significance and regulation. Front. Plant. Sci. 4:118. doi: 10.3389/fpls.2013.00118

Pawar, P. M. A., Ratke, C., Balasubramanian, V. K., Chong, S. L., Gandla, M. L., Adriasola, M., et al. (2017b). Downregulation of RWA genes in hybrid aspen affects xylan acetylation and wood saccharification. New Phytol. 214, 1491-1505. doi: 10.1111/nph.14489

Ponder, G. R., and Richards, G. N. (1997). Arabinogalactan from Western larch, Part III: alkaline degradation revisited, with novel conclusions on molecular structure. Carbohydr. Polym. 34, 251-261. doi: 10.1016/S0144-8617(97)00 099-4

Ratke, C., Terebieniec, B. K., Winestrand, S., Derba-Maceluch, M., Grahn, T., Schiffthaler, B., et al. (2015). Populus GT43 family members group into distinct sets required for primary and secondary wall xylan biosynthesis and 
include useful promoters for wood modification. Plant Biotechnol. J. 13, 26-37. doi: $10.1111 /$ pbi.12232

Ratke, C., Terebieniec, B. K., Winestrand, S., Derba-maceluch, M., Grahn, T., Schiffthaler, B., et al. (2018). Downregulating aspen xylan biosynthetic GT43 genes in developing wood stimulates growth via reprograming of the transcriptome. New Phytol. 219, 230-245. doi: 10.1111/nph.15160

Ruel, K., Chevalier-Billosta, V., Guillemin, F., Sierra, J. B., and Joseleau, J.P. (2006). The wood cell wall at the ultrastructural scale-formation and topochemical organization. Maderas Ciencia Tecnol. 8, 107-116. doi: 10.4067/ S0718-221X2006000200004

Salmén, L. (2015). Wood morphology and properties from molecular perspectives. Ann. For. Sci. 72, 679-684. doi: 10.1007/s13595-014-0403-3

Sandquist, D., Filonova, L., von Schantz, L., Ohlin, M., and Daniel, G. (2010). Microdistribution of xyloglucan in differentiating poplar cells. BioResources 5 , 796-807.

Sawada, D., Kalluri, U. C., O’Neill, H., Urban, V., Langan, P., Davison, B., et al. (2018). Tension wood structure and morphology conducive for better enzymatic digestion. Biotechnol. Biofuels 11:44. doi: 10.1186/s13068-018-1 043-x

Scheller, H. V., and Ulvskov, P. (2010). Hemicelluloses. Annu. Rev. Plant Biol. 61, 263-289. doi: 10.1146/annurev-arplant-042809-112315

Schröder, R., Atkinson, R. G., and Robert, J. (2009). Re-interpreting the role of endo- $\beta$-mannanases as mannan endotransglycosylase/hydrolases in the plant cell wall. Ann. Bot. 104, 197-204. doi: 10.1093/aob/mcp120

Siedlecka, A., Wiklund, S., Péronne, M.-A., Micheli, F., Leśniewska, J., Sethson, I., et al. (2008). Pectin methyl esterase inhibits intrusive and symplastic cell growth in developing wood cells of Populus. Plant Physiol. 146, 554-565. doi: 10.1104/ pp.107.111963

Sjöström, E. (1993). Wood Chemistry - Fundamentals and Applications. San Diego, CA: Academic Press.

Smith, P. J., Wang, H. T., York, W. S., Peña, M. J., and Urbanowicz, B. R. (2017) Designer biomass for next-generation biorefineries: leveraging recent insights into xylan structure and biosynthesis. Biotechnol. Biofuels 10:286. doi: 10.1186/ s13068-017-0973-z

Song, D., Gui, J., Liu, C., Sun, J., and Li, L. (2016). Suppression of PtrDUF579-3 expression causes structural changes of the glucuronoxylan in Populus. Front. Plant Sci. 7:493. doi: 10.3389/fpls.2016.00493

Sun, S., Sun, S., Cao, X., and Su, R. (2016). The role of pretreatment in improving the enzymatic hydrolysis of lignocellulosic materials. Bioresour. Technol. 199, 49-58. doi: 10.1016/j.biortech.2015.08.061

Suzuki, S., Li, L., Sun, Y. H., and Chiang, V. L. (2006). The cellulose synthase gene superfamily and biochemical functions of xylem-specific cellulose synthase-like genes in Populus trichocarpa. Plant Physiol. 142, 1233-1245. doi: 10.1104/pp. 106.086678

Tan, L., Eberhard, S., Pattathil, S., Warder, C., Glushka, J., Yuan, C., et al. (2013). An Arabidopsis cell wall proteoglycan consists of pectin and arabinoxylan covalently linked to an arabinogalactan protein. Plant Cell 25, 270-287. doi: $10.1105 /$ tpc.112.107334

Taniguchi, T., Konagaya, K., Kurita, M., Takata, N., Ishii, K., Kondo, T., et al. (2012). Growth and root sucker ability of field-grown transgenic poplars overexpressing xyloglucanase. J. Wood Sci. 58, 550-556. doi: 10.1007/s100 86-012-1281-7

Tavares, E. Q., De Souza, A. P., and Buckeridge, M. S. (2015). How endogenous plant cell-wall degradation mechanisms can help achieve higher efficiency in saccharification of biomass. J. Exp. Bot. 66, 4133-4143. doi: 10.1093/jxb/erv171
Teleman, A. (2009). "Hemicelluloses and pectins," in Wood Chemistry and Wood Biotechnology, Vol. 1, eds G. Gellerstedt, M. Ek, and G. Henriksson (Berlin: De Gruyter), 101-120.

Teleman, A., Lundqvist, J., Tjerneld, F., Stålbrand, H., and Dahlman, O. (2000). Characterization of acetylated 4-O-methylglucuronoxylan isolated from aspen employing $1 \mathrm{H}$ and 13C NMR spectroscopy. Carbohydr. Res. 329, 807-815. doi: 10.1016/S0008-6215(00)00249-4

Teleman, A., Tenkanen, M., Jacobs, A., and Dahlman, O. (2002). Characterization of O -acetyl- (4- O -methylglucurono) xylan isolated from birch and beech. Carbohydr. Res. 337, 373-377. doi: 10.1016/S0008-6215(01)00327-5

Wang, Y., Fan, C., Hu, H., Li, Y., Sun, D., Wang, Y., et al. (2016). Genetic modification of plant cell walls to enhance biomass yield and biofuel production in bioenergy crops. Biotechnol. Adv. 34, 997-1017. doi: 10.1016/j.biotechadv. 2016.06.001

Wang, Y., Mortimer, J. C., Davis, J., Dupree, P., and Keegstra, K. (2013). Identification of an additional protein involved in mannan Biosynthesis. Plant J. 73, 105-117. doi: 10.1111/tpj.12019

Wang, Z., Wu, G., and Jönsson, L. J. (2018). Effects of impregnation of softwood with sulfuric acid and sulfur dioxide on chemical and physical characteristics, enzymatic digestibility, and fermentability. Bioresour. Technol. 247, 200-208. doi: 10.1016/j.biortech.2017.09.081

Yamamoto, M., Saito, T., Isogai, A., Kurita, M., Kondo, T., Taniguchi, T., et al. (2011). Enlargement of individual cellulose microfibrils in transgenic poplars overexpressing xyloglucanase. J. Wood Sci. 57, 71-75. doi: 10.1007/s10086-0 10-1140-3

Yang, B., and Wyman, C. E. (2008). Pretreatment: the key to unlocking lowcost cellulosic ethanol. Biofuel Bioprod. Biorefin. 2, 26-40. doi: 10.1002/ bbb. 49

Yang, Y., Yoo, C. G., Winkeler, K. A., Collins, C. M., Hinchee, M. A. W., Jawdy, S. S., et al. (2017). Overexpression of a domain of unknown function 231containing protein increases $\mathrm{O}$-xylan acetylation and cellulose biosynthesis in Populus. Biotechnol. Biofuels 10:311. doi: 10.1186/s13068-017-0998-3

Zhang, M., Chavan, R. R., Smith, B. G., McArdle, B. H., and Harris, P. J. (2016). Tracheid cell-wall structures and locations glucans in compression woods of radiata pine (Pinus radiata D. Don). BMC Plant Biol. 16:194. doi: 10.1186/ s12870-016-0884-3

Zhao, W., Odelius, K., Edlund, U., Zhao, C., and Albertsson, A. C. (2015). In situ synthesis of field-responsive hemicellulose hydrogels for drug delivery. Biomacromolecules 16, 2522-2528. doi: 10.1021/acs.biomac.5b00801

Zhao, Y., Zhang, Q., Yuan, L., Zhang, R., and Li, L. (2013). N-glycosylation and dimerization regulate the PtrMAN6 enzyme activity that may modulate generation of oligosaccharide signals. Plant Signal. Behav. 8:e26956. doi: 10. 4161/psb. 26956

Conflict of Interest Statement: The authors declare that the research was conducted in the absence of any commercial or financial relationships that could be construed as a potential conflict of interest.

Copyright (c) 2018 Donev, Gandla, Jönsson and Mellerowicz. This is an open-access article distributed under the terms of the Creative Commons Attribution License (CC BY). The use, distribution or reproduction in other forums is permitted, provided the original author(s) and the copyright owner(s) are credited and that the original publication in this journal is cited, in accordance with accepted academic practice. No use, distribution or reproduction is permitted which does not comply with these terms. 